\title{
EXTREMAL DISTRIBUTIONS OF DISCREPANCY FUNCTIONS
}

\author{
RALPH KRITZINGER AND MARKUS PASSENBRUNNER
}

\begin{abstract}
The irregularities of a distribution of $N$ points in the unit interval are often measured with various notions of discrepancy. The discrepancy function can be defined with respect to intervals of the form $[0, t) \subset[0,1)$ or arbitrary subintervals of the unit interval. In the former case, it is a well known fact in discrepancy theory that the $N$ element point set in the unit interval with the lowest $L_{2}$ or $L_{\infty}$ norm of the discrepancy function is the centered regular grid

$$
\Gamma_{N}:=\left\{\frac{2 n+1}{2 N}: n=0,1, \ldots, N-1\right\} .
$$

We show a stronger result on the distribution of discrepancy functions of point sets in $[0,1]$, which basically says that the distribution of the discrepancy function of $\Gamma_{N}$ is in some sense minimal among all $N$-element point sets. As a consequence, we can extend the above result to rearrangement-invariant norms, including $L_{p}$, Orlicz and Lorentz norms.

We study the same problem for the discrepancy notions with respect to arbitrary subintervals. In this case, we will observe that we have to deal with integrals of convolutions of functions. To this end, we prove a general upper bound on such expressions, which might be of independent interest as well.
\end{abstract}

\section{INTRODUCTION}

Let $\mathcal{P}=\left\{x_{0}, \ldots, x_{N-1}\right\}$ be an $N$-element point set in the unit interval $[0,1]$ which is always assumed to be arranged increasingly. Denote by $D=D_{\mathcal{P}}$ its one-parameter discrepancy function

$$
D(t)=\sum_{n=0}^{N-1} \mathbb{1}_{[0, t)}\left(x_{n}\right)-N t,
$$

where $\mathbb{1}_{A}$ denotes the indicator function of the set $A$. We also consider a two-parameter discrepancy function $\widetilde{D}=\widetilde{D}_{\mathcal{P}}$, defined by

$$
\widetilde{D}\left(t_{1}, t_{2}\right)=\sum_{n=0}^{N-1} \mathbb{1}_{\left[t_{1}, t_{2}\right)}\left(x_{n}\right)-N\left(t_{2}-t_{1}\right), \quad 0 \leq t_{1} \leq t_{2} \leq 1 .
$$

Therefore, the discrepancy functions measure the deviation of the actual number of points in a subinterval of $[0,1]$ (the so-called test sets) and the expected number of points under the assumption of uniform distribution. This deviation is measured with respect to intervals anchored in the origin in the case of $D_{\mathcal{P}}$ and with respect to arbitrary subintervals of $[0,1]$ in the case of $\widetilde{D}_{\mathcal{P}}$.

One usually considers a norm of the discrepancy function as a quantitative measure of the irregularities of distribution of a point set. The best-studied cases are those of the $L_{p}$ norms for $1 \leq p \leq \infty$, where we speak of $L_{p}$ discrepancy for finite $p$ and of star

2010 Mathematics Subject Classification. 11K06, 11K38, 42A85, 46E30.

Key words and phrases. discrepancy function, convolution inequalities. 
discrepancy for $p=\infty$ in case of the one-parameter discrepancy function. Note that for a measurable function $f: A \rightarrow \mathbb{R}$ defined on a domain $A \subseteq \mathbb{R}^{d}$ with $|A|>0$, where $|\cdot|$ denotes the $d$-dimensional Lebesgue measure, we define the $L_{p}$ (quasi-) norm of $f$ for $p \in(0, \infty)$ by

$$
\|f\|_{p}:=\left(\int_{A}|f(t)|^{p} \mathrm{~d} t\right)^{1 / p}
$$

and the $L_{\infty}$ norm by

$$
\|f\|_{\infty}:=\inf \{\lambda \geq 0:|f| \leq \lambda \text { a.e. }\} .
$$

If we take the same norms of the two-parameter discrepancy function, one usually speaks of extreme $L_{p}$ and star discrepancy, respectively. Consult e.g. 3] for an overview on these notions and [9] for an excellent introduction to discrepancy theory. The smaller these discrepancy notions of a point set $\mathcal{P}$, the more uniformly it is distributed in the unit interval (see e.g. 6]). The determination of those $N$-element point sets that have minimal discrepancy is a very difficult and largely unsolved problem in dimensions higher than one. We refer to [13] for the $N$-element point sets in $[0,1]^{2}$ with minimal star discrepancy for $N=1, \ldots, 6$ and to [12] and [7] for the one and two-element point sets in the $d$-dimensional unit cube $[0,1]^{d}$ with minimal $L_{2}$, star and extreme star discrepancy, respectively. Moreover, a systematic search for the $N$-element point sets with minimal $L_{2}$ discrepancy, measured with respect to periodic boxes, up to $N=16$, was performed in [5]. However, for point sets in the one-dimensional unit interval $[0,1]$ the answer is known for the star and $L_{2}$ discrepancy and every natural number $N$. By Niederreiter (see [10, Corollary 1.1] and [11, Theorem 2.6]) we have for the $L_{2}$ and star discrepancy of a set of points $\mathcal{P}=\left\{x_{0}, \ldots, x_{N-1}\right\}$ the explicit formulas

$$
\left\|D_{\mathcal{P}}\right\|_{2}=N \sum_{n=0}^{N-1}\left(x_{n}-\frac{2 n+1}{2 N}\right)^{2}+\frac{1}{12}
$$

and

$$
\left\|D_{\mathcal{P}}\right\|_{\infty}=N \max _{0 \leq n \leq N-1}\left|x_{n}-\frac{2 n+1}{2 N}\right|+\frac{1}{2}
$$

respectively. As an immediate consequence we find that the centered regular grid

$$
\Gamma_{N}:=\left\{\frac{2 n+1}{2 N}: n=0, \ldots, N-1\right\} .
$$

is the unique minimizer of both the $L_{2}$ and star discrepancy among all $N$-element points in $[0,1]$.

The situation is similar for the extreme discrepancy notions. Niederreiter [11, Theorem 2.7] was able to show the explicit formula

$$
\left\|\widetilde{D}_{\mathcal{P}}\right\|_{\infty}=1+N \max _{0 \leq n \leq N-1}\left(\frac{n}{N}-x_{n}\right)-N \min _{0 \leq n \leq N-1}\left(\frac{n}{N}-x_{n}\right)
$$

for $\mathcal{P}=\left\{x_{0}, \ldots x_{N-1}\right\} \subset[0,1]$. Furthermore, it is not hard to prove the following formula for its extreme $L_{2}$ discrepancy. By a straight-forward computation of the integrals in its definition and some elementary algebra we find

$$
\left\|\widetilde{D}_{\mathcal{P}}\right\|_{2}^{2}=\frac{1}{12}+\frac{1}{2} \sum_{n, m=0}^{N-1}\left(x_{n}-x_{m}-\frac{n-m}{N}\right)^{2} .
$$


Therefore, the only minimizing point sets with $N$ elements of the extreme star and $L_{2}$ discrepancy are translated regular grids of the form

$$
\Gamma_{N}^{\delta}=\left\{\frac{n}{N}+\delta: n=0, \ldots, N-1\right\} \text { for some } \delta \in\left[0, \frac{1}{N}\right) .
$$

Observe that with this notation, we have $\Gamma_{N}^{1 /(2 N)}=\Gamma_{N}$.

The question arises whether these statements remain true if we take other norms of the one- and two-parameter discrepancy function of $\mathcal{P}$. To this end, we will show results on the distribution of the discrepancy functions, which is motivated by the fact that the $L_{p}$ norm and various other norms of the discrepancy functions are determined by the distribution of their absolute values. In general, by distribution we mean the following: Let $f: A \rightarrow \mathbb{R}$ be a measurable function on a domain with $A \subseteq \mathbb{R}^{d}$ and $|A|>0$. Then we define $\mathbb{P}_{A}(f<\alpha):=|\{t \in A:|f(t)|<\alpha\}| /|A|$. Here, we denote by $|\cdot|$ the Lebesgue measure on $\mathbb{R}^{d}$. We usually suppress the lower index in $\mathbb{P}_{A}$. Hence, we ask for results on the distributions $\mathbb{P}\left(\left|D_{\mathcal{P}}\right|<\alpha\right)$ and $\mathbb{P}\left(\left|\widetilde{D}_{\mathcal{P}}\right|<\alpha\right)$ of the one- and two-parameter discrepancy function, respectively. To be more precise, we will show that for any natural number $N$ and any point sequence $\mathcal{P}$ with $N$ elements, we have

$$
\mathbb{P}\left(\left|D_{\mathcal{P}}\right|<\alpha\right) \leq \mathbb{P}\left(\left|D_{\Gamma_{N}}\right|<\alpha\right) \quad \text { and } \quad \mathbb{P}\left(\left|\widetilde{D}_{\mathcal{P}}\right|<\alpha\right) \leq \mathbb{P}\left(\left|\widetilde{D}_{\Gamma_{N}}\right|<\alpha\right), \quad \alpha>0,
$$

with equality for all $\alpha>0$ only if $\mathcal{P}$ is the centered regular grid $\Gamma_{N}$ in the first inequality or a translation thereof in the second. Normed function spaces where the norm of a function is determined by its distribution are called rearrangement-invariant and include Orlicz and Lorentz spaces, for instance, which both generalize the $L_{p}$ spaces. As a general reference to those notions, we use [1]. We will use our results on the distribution functions of $D$ and $\widetilde{D}$ to identify the centered regular grid $\Gamma_{N}$ and its translations as the (only) minimizers of each such norm of $D$ and $\widetilde{D}$, respectively.

\section{THE DisTRIBUtion OF THE ONE-PARAMETER DISCREPANCY FUNCTION}

The function $D$ and its extremal distribution are easily analyzed. To this end, we investigate the distribution function $\mathbb{P}(D \leq \alpha):=|\{t \in[0,1]: D(t) \leq \alpha\}|$ of $D=D_{\mathcal{P}}$. We observe that if $\ell$ is a linear function on an interval $I \subset \mathbb{R}$ of finite length that has a slope of $k \neq 0$, we get

$$
|\{x \in I: \ell(x) \in(a, b)\}|=\int_{a}^{b} \frac{\mathbb{1}_{\ell(I)}(t)}{|k|} \mathrm{d} t, \quad a<b .
$$

Let now $g$ denote the density of $D$; i.e. $g \geq 0$ with $\int g=1$ is such that

$$
\mathbb{P}(D \in(a, b))=\int_{a}^{b} g(t) \mathrm{d} t, \quad a<b .
$$

Observe that $D$ consists of $N+1$ linear pieces with slope $-N$ on the intervals $\left(x_{n-1}, x_{n}\right)$ for $n=0, \ldots, N$ with $x_{-1}=0, x_{N}=1$. Therefore, by the above argument and setting $I_{n}=\left(n-N x_{n}, n-N x_{n-1}\right)$ for $n=0, \ldots, N$,

$$
g=\frac{1}{N} \sum_{n=0}^{N} \mathbb{1}_{I_{n}} .
$$


Since $I_{0} \cap I_{N}=\emptyset$, the function $g$ is piecewise constant with $g \in\{j / N: 0 \leq j \leq N\}$. For instance, the density $g$ corresponding to the translated grid $\Gamma_{N}^{\delta}$-as defined in (1.4) - is given by $\mathbb{1}_{[-N \delta, 1-N \delta)}$.

It is easily seen that the properties $0 \leq g \leq 1$ and $\int g=1$ imply that for intervals $I$ symmetric around 0 , we have the inequality

$$
\int_{I} g(t) \mathrm{d} t \leq \int_{I} M_{1}(t) \mathrm{d} t
$$

with $M_{1}=\mathbb{1}_{(-1 / 2,1 / 2)}$. In (2.2), equality for all symmetric intervals $I$ around 0 holds exactly if $g=M_{1}$ a.e. This immediately implies the following result:

Theorem 2.1. For any natural number $N$ and any point sequence $\mathcal{P}$ with $N$ elements, we have

$$
\mathbb{P}\left(\left|D_{\mathcal{P}}\right|<\alpha\right) \leq \mathbb{P}\left(\left|D_{\Gamma_{N}}\right|<\alpha\right), \quad \alpha>0,
$$

and equality for all $\alpha>0$ holds if and only if $\mathcal{P}=\Gamma_{N}$.

Proof. With the density $g$ of $D_{\mathcal{P}}$ introduced above we can write for $\alpha>0$

$$
\mathbb{P}\left(\left|D_{\mathcal{P}}\right|<\alpha\right)=\mathbb{P}\left(D_{\mathcal{P}} \in(-\alpha, \alpha)\right)=\int_{-\alpha}^{\alpha} g(t) \mathrm{d} t \leq \int_{-\alpha}^{\alpha} M_{1}(t) \mathrm{d} t=\mathbb{P}\left(\left|D_{\Gamma_{N}}\right|<\alpha\right)
$$

by (2.2) with equality for all $\alpha>0$ exactly for $g=M_{1}$ a.e., which is the case only if $\mathcal{P}=\Gamma_{N}$.

\section{Convolution inequalities}

In this section, we show a general inequality involving integrals and the convolution of functions with certain properties, which will lead to estimates for the distribution function of $\widetilde{D}$. Before we state these results, we explain the relationship between $\widetilde{D}$ and convolutions of functions. The function $\widetilde{D}$ in (1.2) is only defined on the set $S=\left\{\left(t_{1}, t_{2}\right) \in\right.$ $\left.[0,1]^{2}: t_{1} \leq t_{2}\right\}$ and on that set, it can be written as $\widetilde{D}\left(t_{1}, t_{2}\right)=D\left(t_{2}\right)-D\left(t_{1}\right)$ with $D$ defined as in (1.1). We extend $\widetilde{D}$ to the set $[0,1]^{2} \backslash S$ by the same formula, which implies that $|\widetilde{D}|$ has the same distribution on the set $S$ as it has on $[0,1]^{2}$. In the following, we always consider $\widetilde{D}$ to be defined on $[0,1]^{2}$. Denoting again by $g$ the probability density of $D$, the probability density of $-D$ is given by $\widetilde{g}$ with $\widetilde{g}(x):=g(-x)$ for $x \in \mathbb{R}$. Since the probability density of the sum of independent random variables is the convolution of their densities, we obtain

$$
\mathbb{P}(|\widetilde{D}|<\alpha)=\left|\left\{\left(t_{1}, t_{2}\right) \in[0,1]^{2}: D\left(t_{2}\right)-D\left(t_{1}\right) \in(-\alpha, \alpha)\right\}\right|=\int_{-\alpha}^{\alpha}(g * \widetilde{g})(t) \mathrm{d} t,
$$

with the convolution $f * g$ given by $(f * g)(x)=\int f(x-y) g(y) \mathrm{d} y$. Therefore, we have to work with integrals where the integrands involve convolutions of functions with certain properties like those of $g$ stated above. In the rest of this section, we will prove an upper bound on such integrals.

Let $f: \mathbb{R} \rightarrow[0, \infty]$ be a non-negative function. We say that $f$ is symmetrically decreasing (or short: s.d.), if $f(-x)=f(x)$ for all $x \in \mathbb{R}$ and, for all $0<x<y$, we have $f(y) \leq f(x)$. It is easy to see that each s.d. function $f$ can be approximated from below pointwise a.e. by an increasing sequence of simple functions of the form $\sum_{i} c_{i} \mathbb{1}_{\left(-t_{i}, t_{i}\right)}$ for 
$t_{i} \in[0, \infty]$ and $c_{i} \geq 0$. Characteristic functions of the form $\mathbb{1}_{I}$ for a symmetric interval $I$ around zero are s.d. and the convolution of two such functions is given by

$$
\mathbb{1}_{I} * \mathbb{1}_{J}(x)=|I \cap(x+J)|, \quad x \in \mathbb{R},
$$

which is again s.d. Therefore, by pointwise approximation with simple functions and the monotone convergence theorem, we conclude that the convolution $f * g$ of two arbitrary s.d. functions $f, g$ is again s.d. Now we show that the function $M_{1}=\mathbb{1}_{(-1 / 2,1 / 2)}$ is largest among all s.d. functions $g$ satisfying $0 \leq g \leq 1$ and $\int g=1$ in the following sense:

Theorem 3.1. Let $f, g, h$ be symmetrically decreasing functions on $\mathbb{R}$ with $0 \leq g \leq 1$ and $\int g=1$. Then,

$$
\int h(x)(f * g)(x) \mathrm{d} x \leq \int h(x)\left(f * M_{1}\right)(x) \mathrm{d} x .
$$

Moreover, if $f$ is not constant a.e. and $f * g \in L_{1}$, equality for all $h$ here implies that $g=M_{1}$ (a.e).

Proof. We begin by proving (3.2). Approximating the s.d. function $h$ by simple functions as described above and using the monotone convergence theorem, it suffices to consider $h=\mathbb{1}_{(-\alpha, \alpha)}$ with $\alpha>0$. Observe that by Fubini's theorem

$$
\int_{-\alpha}^{\alpha}(f * g)(t) \mathrm{d} t=\int f(z) \int_{-\alpha}^{\alpha} g(t-z) \mathrm{d} t \mathrm{~d} z .
$$

Let $v(z)=v_{\alpha}(z)=\left(\mathbb{1}_{(-\alpha, \alpha)} * g\right)(z)=\int_{-\alpha}^{\alpha} g(t-z) \mathrm{d} t=\int_{-\alpha-z}^{\alpha-z} g(t) \mathrm{d} t$. Note that $v$, as the convolution of two s.d. functions, is s.d. We next show the following properties of $v$ :

(1) $0 \leq v \leq \min (2 \alpha, 1)$,

(2) $\int v=2 \alpha$,

(3) $v$ is 1-Lipschitz.

Since $0 \leq g \leq 1$ and $\int g=1$, we have $0 \leq v \leq \min (2 \alpha, 1)$, showing property (1). For property (2), we just calculate

$$
\int v(z) \mathrm{d} z=\int_{-\alpha}^{\alpha} \int g(t-z) \mathrm{d} z \mathrm{~d} t=2 \alpha
$$

where in the last equality, we used $\int g=1$. Finally, property (3) is seen by the fact that $v(z)=\int_{-\alpha-z}^{\alpha-z} g(t) \mathrm{d} t$ implies that, for $\rho>0, v(z+\rho)-v(z)$ can be written as

$$
v(z+\rho)-v(z)=\int_{I} g(t) \mathrm{d} t-\int_{J} g(t) \mathrm{d} t
$$

for two intervals $I, J$ with $|I| \leq \rho$ and $|J| \leq \rho$. Therefore, the assumption $0 \leq g \leq 1$ implies $|v(z+\rho)-v(z)| \leq \rho$, which is (3).

Next, define $v_{0}=v_{0, \alpha}=\mathbb{1}_{(-\alpha, \alpha)} * M_{1}$. Then, $v_{0}$ is the s.d. function that equals $\min (2 \alpha, 1)$ on $[0,|\alpha-1 / 2|]$, has slope -1 on $(|\alpha-1 / 2|, \alpha+1 / 2)$ and equals zero on $[\alpha+1 / 2, \infty)$. By the properties (1) and (2) of $v$ and the definition of $v_{0}$, for $t_{0}>0$ with $t_{0}<|\alpha-1 / 2|$ or $t_{0}>\alpha+1 / 2$, we clearly have

$$
\int_{-t_{0}}^{t_{0}} v(z) \mathrm{d} z \leq \int_{-t_{0}}^{t_{0}} v_{0}(z) \mathrm{d} z .
$$

If $|\alpha-1 / 2| \leq t_{0} \leq \alpha+1 / 2$, we distinguish the cases $v\left(t_{0}\right) \leq v_{0}\left(t_{0}\right)$ and $v\left(t_{0}\right)>v_{0}\left(t_{0}\right)$. In the first case, by (3), (1) for $v$ and the definition of $v_{0}$, we have (3.3). In the second case, 
by the same reasoning, $\int_{t_{0}}^{\infty} v(z) \mathrm{d} z \geq \int_{t_{0}}^{\infty} v_{0}(z) \mathrm{d} z$, which, using property (2) for $v$ and $v_{0}$ also yields (3.3). By approximating the s.d. function $f$ as above by an increasing sequence of simple functions and using the monotone convergence theorem, inequality (3.3) implies

$$
\int_{-\alpha}^{\alpha}(f * g)(t) \mathrm{d} t=\int f(z) v(z) \mathrm{d} z \leq \int f(z) v_{0}(z) \mathrm{d} z=\int_{-\alpha}^{\alpha}\left(f * M_{1}\right)(z) \mathrm{d} z,
$$

which concludes the proof of inequality (3.2).

Now, we prove the equality part. We assume that $f$ is not constant a.e. and $g \neq M_{1}$. This implies that $a:=\operatorname{essinf} f<\operatorname{ess} \sup f=: b$ and the existence of $x_{0} \in(0,1 / 2)$ so that $g(t)<1$ for all $t \in\left(x_{0}, 1 / 2\right)$. Set $\delta=1 / 2-x_{0}>0$.

First, we show that for $\alpha \geq 0$ and $I_{\alpha}=\left(\alpha-1 / 2, \alpha-x_{0}\right)$, we have the inequality

$$
v_{\alpha}(z)<v_{0, \alpha}(z), \quad z \in I_{\alpha} \cup\left(-I_{\alpha}\right)
$$

Indeed, for $z \in I_{\alpha}$, we decompose the set $(-\alpha-z, \alpha-z)$ into the two intervals $J_{1}:=$ $\left(\min \left(-\alpha-z, x_{0}\right), x_{0}\right)$ and $J_{2}:=\left(\max \left(x_{0},-\alpha-z\right), \alpha-z\right) \subset\left(x_{0}, 1 / 2\right)$, with $\left|J_{2}\right|>0$. By the properties of $g$ and $M_{1}$, the inequality $\int_{J_{1}} g(t) \mathrm{d} t \leq \int_{J_{1}} M_{1}(t) \mathrm{d} t$ is true. Moreover, for $t \in J_{2}$, we have $g(t)<1=M_{1}(t)$. The fact that $\left|J_{2}\right|>0$ then implies $\int_{J_{2}} g(t) \mathrm{d} t<$ $\int_{J_{2}} M_{1}(t) \mathrm{d} t$. Since $v(z)=\int_{J_{1}} g(t) \mathrm{d} t+\int_{J_{2}} g(t) \mathrm{d} t$ and $v_{0}(z)=\int_{J_{1}} M_{1}(t) \mathrm{d} t+\int_{J_{2}} M_{1}(t) \mathrm{d} t$, adding up the above two inequalities yields (3.5) for $z \in I_{\alpha}$. Since the functions $v$ and $v_{0}$ are both s.d., (3.5) also holds for $z \in-I_{\alpha}$.

Next, we choose the parameters $s_{1}, s_{2}$ with $a<s_{1}<s_{2}<b, \eta \in(0, \delta / 2)$ and $t_{0}>0$ in such a way that

- $t_{0}-\eta>0$,

- $f(t) \leq s_{1}$ if $t \geq t_{0}+\eta$,

- $f(t) \geq s_{2}$ if $0 \leq t \leq t_{0}$.

This is possible because, due to the continuity of the Lebesgue measure, the measure of the set $\left\{f \in\left(s_{1}, s_{2}\right)\right\}$ can be chosen arbitrarily small if $s_{2}-s_{1}$ is sufficiently small.

Define $U=\left(t_{0}-\eta, t_{0}+\eta\right) \cup\left(-t_{0}-\eta,-t_{0}+\eta\right)$ and decompose $f=f_{1}+f_{2}$ with

$$
f_{1}=\left(\min \left(f, s_{2}\right)-s_{1}\right) \cdot \mathbb{1}_{\left\{f \geq s_{1}\right\}} \cdot \mathbb{1}_{U}, \quad f_{2}=f-f_{1} .
$$

Observe that $f_{2}$ is s.d. and $f_{1} \geq 0$. Since $\left|\left(t_{0}-\eta, t_{0}+\eta\right)\right|=2 \eta \leq \delta=\left|I_{\alpha}\right|$ for all $\alpha$, we can choose $\alpha>0$ so that $U \subseteq I_{\alpha} \cup\left(-I_{\alpha}\right)$. Doing so, (3.4) implies $\int f_{2}(z) v_{\alpha}(z) \mathrm{d} z \leq$ $\int f_{2}(z) v_{0, \alpha}(z) \mathrm{d} z$ and (3.5) implies $\int f_{1}(z) v_{\alpha}(z) \mathrm{d} z<\int f_{1}(z) v_{0, \alpha}(z) \mathrm{d} z$, where in the strict inequality, we also use the fact that $f_{1}=s_{2}-s_{1}>0$ on a subset of $U$ having positive Lebesgue measure. Adding up those inequalities, we conclude (using that $f * g \in L_{1}$ )

$$
\int_{-\alpha}^{\alpha}(f * g)(t) \mathrm{d} t=\int f(z) v_{\alpha}(z) \mathrm{d} z<\int f(z) v_{0, \alpha}(z) \mathrm{d} z=\int_{-\alpha}^{\alpha}\left(f * M_{1}\right)(t) \mathrm{d} t
$$

for $\alpha>0$ chosen above, which finishes the proof of the equality part.

We next extend the result in Theorem 3.1 to more general functions and arbitrarily many convolution factors. To this end, we need an important classical inequality involving convolutions and symmetric rearrangements, which we now describe. Two functions $f, g$ : $\mathbb{R} \rightarrow[0, \infty]$ are equimeasurable, if the level sets $\{f \geq \lambda\}$ and $\{g \geq \lambda\}$ for $\lambda \geq 0$ have the same Lebesgue measure. The symmetric decreasing rearrangement of a non-negative function $f$ is given by the s.d. function $f^{*}$ that is equimeasurable with $f$. The symmetric decreasing rearrangement is uniquely determined up to equality almost everywhere. The 
following inequality is known for $n=1$ as the Hardy-Littlewood inequality, for $n=2$ as the Riesz inequality and for $n>2$ it is due to Luttinger and Friedberg [8]

$$
\int f(x)\left(g_{1} * \cdots * g_{n}\right)(x) \mathrm{d} x \leq \int f^{*}(x)\left(g_{1}^{*} * \cdots * g_{n}^{*}\right)(x) \mathrm{d} x,
$$

where $f, g_{1}, \ldots, g_{n}$ are arbitrary non-negative functions. For an even more general version of this inequality, we refer to [2]. Symmetrically decreasing rearrangements of functions, as well as the above inequality for $n=1,2$ are treated in the classical book Inequalities [4] by Hardy, Littlewood and Pólya. We now define iterative convolutions of the function $M_{1}=\mathbb{1}_{(-1 / 2,1 / 2)}$ as follows:

$$
M_{n}=M_{n-1} * M_{1}, \quad n \geq 2 .
$$

The function $M_{n}$ is known as the centered cardinal B-spline of order $n$.

Combining Theorem 3.1 with inequality (3.6) yields the following convolution inequality which will be used later to estimate the measure of level sets of the extreme discrepancy $\widetilde{D}$ and may be of independent interest:

Theorem 3.2. Let $g_{1}, \ldots, g_{n}$ be functions on $\mathbb{R}$ so that $0 \leq g_{j} \leq 1$ and $\int g_{j}=1$ for all $1 \leq j \leq n$. Then, we have for all non-negative functions $h$

$$
\int h(x)\left(g_{1} * \cdots * g_{n}\right)(x) \mathrm{d} x \leq \int h^{*}(x) M_{n}(x) \mathrm{d} x .
$$

If equality holds for all s.d. functions $h$, it follows that $g_{1}^{*}=\cdots=g_{n}^{*}=M_{1}$ a.e.

Proof. Applying (3.6) to the left hand side of (3.7), we only have to estimate the expression

$$
\int h^{*}(x)\left(g_{1}^{*} * \cdots * g_{n}^{*}\right)(x) \mathrm{d} x .
$$

If $n=1$, this is trivially estimated by $\int h^{*}(x) M_{1}(x) \mathrm{d} x$. For $n \geq 2$, we apply Theorem 3.1 iteratively and use that the convolution of s.d. functions is again s.d. to deduce

$$
\int h^{*}(x)\left(g_{1}^{*} * \cdots * g_{n}^{*}\right)(x) \mathrm{d} x \leq \int h^{*}(x)\left(M_{1} * \cdots * M_{1}\right)(x) \mathrm{d} x=\int h^{*}(x) M_{n}(x) \mathrm{d} x .
$$

If equality holds in (3.7) (for all s.d. functions $h$ ) then, in particular, equality holds in (3.8). By the second part of Theorem 3.1, this is the case if and only if $g_{1}^{*}=\cdots=g_{n}^{*}=M_{1}$ a.e.

\section{MAin RESUlts AND CONSEQUENCES}

We now use the techniques of Section 3 to show a similar result for the extreme discrepancy $\widetilde{D}$ in Theorem 4.1 below as we did for $D$ in Theorem 2.1. Then we calculate various minimal values of rearrangement invariant norms of discrepancy functions in order to demonstrate how to apply the above theorems. The list of norms we consider here is by no means exhaustive, but Theorems 2.1 and 4.1 allow us to treat any desired rearrangement invariant norm.

Theorem 4.1. We have for all $N$-element point sets $\mathcal{P}$,

$$
\mathbb{P}\left(\left|\widetilde{D}_{\mathcal{P}}\right|<\alpha\right) \leq \mathbb{P}\left(\left|\widetilde{D}_{\Gamma_{N}}\right|<\alpha\right), \quad \alpha>0,
$$

and equality for all $\alpha>0$ holds if and only if $\mathcal{P}=\Gamma_{N}^{\delta}$ for some $\delta \in[0,1 / N)$. 
Proof. By (3.1), we can write

$$
\mathbb{P}(|\widetilde{D}|<\alpha)=\int_{-\alpha}^{\alpha}(g * \widetilde{g})(t) \mathrm{d} t
$$

with $g$ being (in particular) a function with $0 \leq g \leq 1$ and $\int g=1$. Now the claim follows from Theorem 3.2 for $n=2$ with $h=\mathbb{1}_{(-\alpha, \alpha)}, g_{1}=g$ and $g_{2}=\widetilde{g}$, which yields

$$
\mathbb{P}\left(\left|\widetilde{D}_{\mathcal{P}}\right|<\alpha\right) \leq \int_{-\alpha}^{\alpha} M_{2}(t) \mathrm{d} t=\mathbb{P}\left(\left|\widetilde{D}_{\Gamma_{N}}\right|<\alpha\right), \quad \alpha>0 .
$$

Equality (for all $\alpha>0$ ) in Theorem 3.2 holds only for $g^{*}=\widetilde{g}^{*}=M_{1}$. Recall that $g$ is of the form (2.1), i.e.,

$$
g=\frac{1}{N} \sum_{n=0}^{N} \mathbb{1}_{I_{n}}
$$

with $I_{n}=\left(n-N x_{n}, n-N x_{n-1}\right)$ for $n=0, \ldots, N$ and $x_{-1}=0, x_{N}=1$. Therefore, the condition $g^{*}=M_{1}$ implies $I_{1}=\cdots=I_{N-1}=I_{0} \cup I_{N}$, which gives $g=\mathbb{1}_{I_{1}}$. But this is only the case for the density of $D_{\Gamma_{N}^{\delta}}$ for any $\delta \in[0,1 / N)$.

Let $\psi:[0, \infty) \rightarrow[0, \infty)$ be an absolutely continuous, strictly increasing function with $\psi(0)=0$. Then, $\psi$ is differentiable a.e. and $\psi(a)=\int_{0}^{a} \psi^{\prime}(s) \mathrm{d} s$ for all $a \geq 0$. Consider a function $f: A \rightarrow \mathbb{R}$, where $A \subseteq \mathbb{R}^{d}$ with $|A|=1$. We define

$$
\|f\|_{\psi}:=\inf \left\{K>0: \int_{A} \psi\left(\frac{|f(t)|}{K}\right) \mathrm{d} t \leq 1\right\}
$$

with the usual convention $\inf \emptyset=\infty$. Note that $\|f\|_{\psi}$ matches the $L_{p}$ (quasi-)norm for $p \in(0, \infty)$ by choosing for $\psi$ the particular function $\psi_{p}:[0, \infty) \rightarrow[0, \infty), s \mapsto s^{p}$. For convex functions $\psi$ in general, $\|f\|_{\psi}$ yields the Orlicz norm. However, the results in this paragraph hold for all functions $\psi$ with the less restrictive properties as stated above. Using Fubini's theorem, we perform the following short and well-known trick introducing the distribution function of $f$ to obtain

$$
\int_{A} \psi\left(\frac{|f(t)|}{K}\right) \mathrm{d} t=\int_{A} \int_{0}^{|f(t)| / K} \psi^{\prime}(\alpha) \mathrm{d} \alpha \mathrm{d} t=\int_{0}^{\infty} \psi^{\prime}(\alpha) \mathbb{P}(|f| \geq K \alpha) \mathrm{d} \alpha .
$$

It is easy to see that for all $\alpha \geq 0$ we have $\mathbb{P}\left(\left|D_{\Gamma_{N}}\right| \geq \alpha\right)=\max \{0,1-2 \alpha\}$ and $\mathbb{P}\left(\left|\widetilde{D}_{\Gamma_{N}}\right| \geq\right.$ $\alpha)=(1-\min \{\alpha, 1\})^{2}$. Define the functions $\Psi, T:[0, \infty) \rightarrow[0, \infty)$ such that $\Psi(0)=$ $T(0)=0$ and $\Psi^{\prime} \equiv \psi, T^{\prime} \equiv \Psi$. Inserting $D$ and $\widetilde{D}$ instead of $f$ in (4.1), Theorem 2.1 and Theorem 4.1 yield

$$
\int_{0}^{1} \psi\left(\frac{\left|D_{\mathcal{P}}(t)\right|}{K}\right) \mathrm{d} t \geq \int_{0}^{1 /(2 K)} \psi^{\prime}(\alpha)(1-2 K \alpha) \mathrm{d} \alpha=2 K \Psi\left(\frac{1}{2 K}\right)
$$

and

$$
\int_{0}^{1} \int_{0}^{1} \psi\left(\frac{\left|\widetilde{D}_{\mathcal{P}}\left(t_{1}, t_{2}\right)\right|}{K}\right) \mathrm{d} t_{2} \mathrm{~d} t_{1} \geq \int_{0}^{1 / K} \psi^{\prime}(\alpha)(1-K \alpha)^{2} \mathrm{~d} \alpha=2 K^{2} T\left(\frac{1}{K}\right)
$$

for every $N$-element point set $\mathcal{P}$ in the unit interval, respectively. As a result, we obtain the following corollary. 
Corollary 4.2. Let $\psi:[0, \infty) \rightarrow[0, \infty)$ be an absolutely continuous, strictly increasing function with $\psi(s)=0$ and $\Psi, T$ as above. Moreover, let $N$ be a non-negative integer.

Then we have

$$
\inf _{\# \mathcal{P}=N}\left\|D_{\mathcal{P}}\right\|_{\psi}=\inf \left\{K>0: 2 K \Psi\left(\frac{1}{2 K}\right) \leq 1\right\}
$$

and

$$
\inf _{\# \mathcal{P}=N}\left\|\widetilde{D}_{\mathcal{P}}\right\|_{\psi}=\inf \left\{K>0: 2 K^{2} T\left(\frac{1}{K}\right) \leq 1\right\},
$$

where the infimum is extended over all $N$-element point sets in $[0,1]$.

Remark 4.3. The special choice $\psi=\psi_{p}: s \mapsto s^{p}$ in (4.2) and (4.3) yields

$$
\inf _{\# \mathcal{P}=N}\left\|D_{\mathcal{P}}\right\|_{p}^{p}=\frac{1}{2^{p}(p+1)} \quad \text { and } \quad \inf _{\# \mathcal{P}=N}\left\|\widetilde{D}_{\mathcal{P}}\right\|_{p}^{p}=\frac{2}{(p+1)(p+2)}
$$

for all $p \in(0, \infty)$. This formula for $\widetilde{D}_{\mathcal{P}}$ with $p=2$ and (1.3) for $\mathcal{P}=\Gamma_{N}$ are different by a factor of 2 , because we consider $\widetilde{D}_{\mathcal{P}}$ to be defined on $[0,1]^{2}$, whereas in formula (1.3) it is integrated over the set $\left\{\left(t_{1}, t_{2}\right) \in[0,1]^{2}: 0 \leq t_{1} \leq t_{2} \leq 1\right\}$.

Finally, we consider Lorentz norms. Let $f$ be a Lebesgue measurable function and $0<p, q<\infty$. We define the Lorentz norm

$$
\|f\|_{p, q}:=p^{1 / q}\left(\int_{0}^{\infty} \alpha^{q-1} \mathbb{P}(|f| \geq \alpha)^{p / q} \mathrm{~d} \alpha\right)^{1 / q} .
$$

Theorem 2.1 and Theorem 4.1 then yield the following lower bounds on Lorentz norms.

Corollary 4.4. Let $B(x, y):=\int_{0}^{1} t^{x-1}(1-t)^{y-1} \mathrm{~d} t$ for $x, y>0$ be the Beta function, $0<p, q<\infty$ and $N$ a non-negative integer.

Then we have

$$
\inf _{\# \mathcal{P}=N}\left\|D_{\mathcal{P}}\right\|_{p, q}^{q}=\frac{p}{2^{q}} B(q, 1+q / p)
$$

and

$$
\inf _{\# \mathcal{P}=N}\left\|\widetilde{D}_{\mathcal{P}}\right\|_{p, q}^{q}=p B(q, 1+2 q / p)
$$

Remark 4.5. Observe that all norms we considered in this section are defined by integrals, where the integrands include the distribution of the discrepancy functions. Let $\mathcal{P}$ be an $N$-element point set with $\mathcal{P} \neq \Gamma_{N}$. Then, since the function $\alpha \mapsto \mathbb{P}\left(\left|D_{\mathcal{P}}\right| \geq \alpha\right)$ is continuous, Theorem 2.1 yields that there is an interval $I$ of positive length such that $\mathbb{P}\left(\left|D_{\mathcal{P}}\right| \geq \alpha\right)>\mathbb{P}\left(\left|D_{\Gamma_{N}}\right| \geq \alpha\right)$ for all $\alpha \in I$. Therefore, equality in (4.2) and (4.4) holds only for $\mathcal{P}=\Gamma_{N}$. With an analogue argumentation and referring to Theorem 4.1 instead of Theorem 2.1, we find that equality in (4.3) and (4.5) holds only for $\mathcal{P}=\Gamma_{N}^{\delta}$ for any $\delta \in[0,1 / N)$. 


\section{OutLOOK}

Considering the assertions of Theorems 2.1 and 4.1, one might wonder whether the fact that for any number of points $N$ there exists a point set $\mathcal{P}^{\prime}$ with $\left|\mathcal{P}^{\prime}\right|=N$ so that for all points sets $\mathcal{P}$ with $|\mathcal{P}|=N$,

$$
\mathbb{P}\left(\left|D_{\mathcal{P}}\right|<\alpha\right) \leq \mathbb{P}\left(\left|D_{\mathcal{P}^{\prime}}\right|<\alpha\right), \quad \alpha>0,
$$

extends to higher dimensions $d \geq 2$. Numerical calculations suggest that such a general result is not true for $d=2$ and $N \geq 4$. Moreover, for $d \geq 3$ and even $N=1$ and $N=2$ such a general result is not true. This was proved for $N=1$ in [12] and for $N=2$ in [7], by showing that the unique $N$-element point sets that minimize $L_{2}$ discrepancy and star discrepancy are different from each other.

A different problem as posed above would be the following: for $d \geq 2$ and any nonnegative integer $N$ find a function $f_{N}$ with the properties:

(1) It satisfies the inequality

$$
\mathbb{P}\left(\left|D_{\mathcal{P}}\right|<\alpha\right) \leq f_{N}(\alpha), \quad \alpha>0
$$

for all point sets $\mathcal{P}$ with $|\mathcal{P}|=N$.

(2) It allows us to give sharp lower bounds for the (quasi-)norm of the discrepancy function in certain function spaces, for instance $L_{1}$ or $L_{p}, p<1$.

The problem of finding such functions $f_{N}$, at least in special cases, will be investigated in the future.

Acknowledgments. R. Kritzinger is supported by the Austrian Science Fund (FWF), Project F5509-N26, and M. Passenbrunner is supported by the Austrian Science Fund (FWF), Project F5513-N26. Both projects are a part of the Special Research Program "Quasi-Monte Carlo Methods: Theory and Applications".

\section{REFERENCES}

[1] C. Bennett and R. Sharpley. Interpolation of operators, volume 129 of Pure and Applied Mathematics. Academic Press, Inc., Boston, MA, 1988.

[2] H. J. Brascamp, E. H. Lieb, and J. M. Luttinger. A general rearrangement inequality for multiple integrals. J. Functional Analysis, 17:227-237, 1974.

[3] C. Doerr, M. Gnewuch, and W. Wahlström. Calculation of discrepancy measures and applications. In W. Chen, A. Srivastav, and G. Travaglini, editors, A Panorama of Discrepancy Theory, pages 621-674. Springer-Verlag, 2014.

[4] G. H. Hardy, J. E. Littlewood, and G. Pólya. Inequalities. Cambridge, at the University Press, 1952. $2 \mathrm{~d}$ ed.

[5] A. Hinrichs and J. Oettershagen. Optimal point sets for quasi-monte carlo integration of bivariate periodic functions with bounded mixed smoothness. Springer Proc. Math. Stat., 163:385-405, 2016.

[6] L. Kuipers and H. Niederreiter. Uniform Distribution of Sequences. Courier Corporation, New York, 1974.

[7] G. Larcher and F. Pillichshammer. A note on optimal point distributions in $[0,1)^{s}$. J. of Computational and Applied Mathematics, 206:977-985, 2007.

[8] J. M. Luttinger and R. Friedberg. A new rearrangement inequality for multiple integrals. Arch. Rational Mech. Anal., 61(1):45-64, 1976.

[9] J. Matoušek. Geometric discrepancy. An illustrated guide, volume 18 of Algorithms and Combinatoriks. Springer-Verlag, Berlin, 1999. 2d ed.

[10] H. Niederreiter. Discrepancy and convex programming. In C.F. Osgood, editor, Diophantine Approximation and Its Applications, pages 129-199. Academic Press, New York, 1973. 
[11] H. Niederreiter. Random Number Generation and Quasi-Monte Carlo Methods. SIAM, Philadelphia, 1992.

[12] T. Pillards, B. Vandewoestyne, and R. Cools. Minimizing the $L_{2}$ and $L_{\infty}$ star discrepancies of a single point in the unit hypercube. J. Comput. Appl. Math., 197(1):282-285, 2006.

[13] B.E. White. On optimal extreme-discrepancy point sets in the square. Numerische Mathematik, $27: 157-164,1977$.

Institute of Financial Mathematics and Applied Number Theory, Johannes Kepler University Linz, Austria, 4040 Linz, Altenberger Strasse 69

E-mail address: ralph.kritzinger@jku.at

Institute of Analysis, Johannes Kepler University Linz, Austria, 4040 Linz, AltenBERGer Strasse 69

E-mail address: markus.passenbrunner@jku . at 